\title{
La déminéralisation par électrodialyse du lactosérum doux de fromagerie
}

\author{
par \\ R. DELBEKE \\ Groupe de travail pour l'amélioration de la qualité des produits laitiers \\ Station Laitière de l'Etat, Melle \\ Centre de Recherches Agronomiques de l'Etat, Gand (Belgique)
}

\section{I. - INTRODUCTION}

Dans beaucoup de pays on perd une grande partie des lactosérums acides (entre autres ceux provenant de la fabrication de fromages frais et de caséine acide) et même une partie des lactosérums doux n'est pas récupérée dans certains pays.

On peut prévoir que le déversement de ces effluents sera bientôt et définitivement interdit par les règlements, de plus en plus sévères, sur la pollution des cours d'eau.

La composition même du lactosérum en restreint les possibilités d'utilisation et le marché mondial de la poudre de lactosérum n'est pas particulièrement stable. L'offre de poudre de lactosérum augmentera à mesure que la surveillance de la pollution deviendra plus rigoureuse et ceci aura évidemment une influence défavorable sur le prix.

Le marché du lactose aussi est à peu près saturé et ne sera pas immédiatement en mesure d'absorber une forte augmentation de la production.

Il faut donc chercher d'autres moyens de valorisation si l'on veut écouler une grande partie du lactosérum en excédent. La déminéralisation est un de ces moyens et la demande en poudre de lactosérum déminéralisée augmente. Il existe aussi un marché en extension pour les concentrés de protéines lactosériques.

La Station Laitière de l'Etat à Melle étudie les possibilités des plus récentes techniques de valorisation, c'est-à-dire de l'électrodialyse et de l'échange d'ions pour la déminéralisation et de la filtration sur gel et de l'ultrafiltration pour la fabrication de concentrés de protéines. Tout en étudiant les possibilités de ces techniques, la Station accorde aussi son attention aux problèmes connexes et à 
l'influence des principaux paramètres et cherche à évaluer grosso modo le coût des différentes opérations.

Nous analyserons ci-après les données recueillies au cours de la déminéralisation de lactosérum doux, provenant de la fabrication de fromage Gouda, par électrolyse à l'aide d'un module d'une marque déterminée. Un exposé des avantages et des inconvénients respectifs de certains systèmes d'électrodialyse ou de ceux de l'électrodialyse et de l'échange d'ions n'est pas envisagé ici.

\section{II. - MODULE ET MEMBRANES}

Les essais ont été effectués à l'aide d'un module de type WD 6/2 de la firme néerlandaise Werkspoor Water équipé de membranes Selemion, des types CMV et AMV, de la firme japonaise Asahi Glass.

Chaque membrane a une surface totale de $0,12 \mathrm{~m}^{2}(60 \mathrm{~cm} \times 20 \mathrm{~cm})$ et une surface effective de $0,0775 \mathrm{~m}^{2}$.

Le nombre de paires de cellules de l'exécution standard s'élève à 50 , ce qui porte la surface effective totale à $3,875 \mathrm{~m}^{2}$. Le nombre de paires de cellules par module peut être augmenté dans une mesure limitée.

La section transversale du circuit hydraulique a une superficie de $2,21 \mathrm{~cm}^{2}(0,13 \mathrm{~cm} \times 17 \mathrm{~cm})$.

\section{III. - CONDITIONS DE TRAVAIL AU COURS DES ESSAIS}

\subsection{Surface effective des membranes}

Le module a été équipé, pour les essais, de 12 paires de cellules totalisant $0,93 \mathrm{~m}^{2}$ de surface effective.

Les membranes étaient disposées de façon à former deux cellules à concentré entre chacune des deux cellules à électrodes et les premières cellules à diluat. Certains constructeurs recommandent cet agencement afin de protéger les électrodes.

\subsection{Circuits hydrauliques}

Nous avons employé un lactosérum concentré, à 25 p. 100 d'extrait sec, obtenu au cours de la fabrication de fromage Gouda (mélange du premier et du deuxième sérums). Un batch de 15 I a été traité à $45^{\circ} \mathrm{C}$ au cours de chaque essai, jusqu'à élimination de 70 p. 100 ou de 90 p. 100 de la quantité initiale de cendre. La vitesse linéaire du 
lactosérum dans les cellules à diluat était de $10 \mathrm{~cm} / \mathrm{s}$ et la chute de pression dans le compartiment à lactosérum du paquet de membranes de $0,25 \mathrm{~kg} / \mathrm{cm}^{2}$. Nous n'avons pas employé d'acides ou de bases pour modifier le $\mathrm{pH}$ du lactosérum avant ou pendant les essais.

Au départ, le concentré consistait en un batch de 151 d'une solution à 1,3 p. 100 de $\mathrm{NaCl}$ acidifiée au $\mathrm{HCl}$ jusqu'au $\mathrm{pH} 3$ et d'une conductivité de $22 \mathrm{mS}$. Nous avons laissé la conductivité du concentré s'élever jusqu'à $60 \mathrm{mS}$, puis maintenu constants le volume, le $\mathrm{pH}$ et la conductivité.

Pour laver les électrodes nous avons employé un batch de 151 d'une solution à $0,1 \mathrm{~N}$ de $\mathrm{Na}_{2} \mathrm{SO}_{4}$ acidifiée au $\mathrm{H}_{2} \mathrm{SO}_{4}$ jusqu'au pH 2,5. Le $\mathrm{pH}$ restait constant pendant l'essai.

Le concentré et le liquide de lavage des électrodes n'étaient ni chauffés, ni refroidis pendant l'essai.

Le lactosérum, le concentré et le liquide de lavage des électrodes coulaient dans le même sens à travers le paquet de membranes ( co-current »). L'alimentation des cellules à électrodes s'accomplissait en parallèle.

La pression dans le compartiment à lactosérum et les cellules à électrodes était plus élevée de $0,02 \mathrm{~kg} / \mathrm{cm}^{2}$ que celle régnant dans le compartiment à concentré.

\subsection{Tension et densité de courant}

La densité de courant a eté maintenue à $25 \mathrm{~mA} / \mathrm{cm}^{2}$ jusqu'à ce que l'on puisse régler la tension à $2,5 \mathrm{~V}$ par paire de cellules.

A partir de ce moment (quelques minutes après le début de chaque essai) la tension restait constante, de sorte que la densité de courant diminuait à mesure qu'augmentait la résistance du module.

\subsection{Schéma de nettoyage}

Le compartiment à lactosérum et le compartiment à concentré étaient nettoyés simultanément après chaque essai, à l'aide de batches de 101 d'eau de puits ordinaire et de solutions à $0,1 \mathrm{~N}$ de $\mathrm{HCl}$ et de $\mathrm{NaOH}$ : $10 \mathrm{mn}$ à l'eau, $20 \mathrm{mn}$ au $\mathrm{HCl}, 10 \mathrm{mn}$ à l'eau, $20 \mathrm{mn}$ au $\mathrm{NaOH}, 10 \mathrm{mn}$ à l'eau, $20 \mathrm{mn}$ au $\mathrm{HCl}$ et $10 \mathrm{mn}$ à l'eau.

On procédait ensuite à un rinçage à l'eau, en continu, jusqu'à ce que le $\mathrm{pH}$ de l'eau dépasse 5.

\section{IV. - METHODES D'ANALYSE}

La densité (masse spécifique, $\mathrm{d}_{4}^{20}{ }^{\circ} \mathrm{C}$ ) a été déterminée à l'aide d'un pycnomètre. 
La présence éventuelle de composants formant des précipités a été recherchée par centrifugation, pendant $15 \mathrm{mn}$ à $40^{\circ} \mathrm{C}$ et à $1200 \mathrm{t} / \mathrm{mn}$ dans un centrifugeur Gerber, de $25 \mathrm{ml}$ de lactosérum contenu dans des tubes de sédimentation à base conique graduée ; une deuxième centrifugation était parfois effectuée après dilution. Le nombre de $\mathrm{ml}$ de sédiment était multiplié par 4 pour permettre l'expression de la quantité de sédiment en $\mathrm{ml}$ par $100 \mathrm{ml}$ de lactosérum.

La détermination des teneurs en extrait sec, en cendre, en azote total, en acide citrique, en chlorures et en phosphore étaiı basée sur les méthodes IDF (1962, 1964, 1962 a, 1967, 1961, 1967 a).

L'azote non protéique a été déterminé par la méthode décrite par De Vleeschauwer et al. (1948).

La teneur en lactose a été déterminée par la méthode colorimétrique de Folin-Wu (longueur d'onde $580 \mathrm{~nm}$ ), telle qu'elle a été décrite par Triebold et Aurand (1963), et les teneurs en Na, en K et en Ca par photométrie de flamme selon la méthode de Deijs et Kowsoleea (1967).

\section{V. - DISCUSSION DES RESULTATS}

\subsection{Données relatives à l'électrodialyse}

Les principales données relatives à l'électrodialyse sont rassemblées dans le tableau 1.

Il s'agit des valeurs moyennes de deux séries d'essais visant à éliminer respectivement 70 p. 100 et 90 p. 100 de la cendre initialement présente. Les données individuelles des essais ne s'écartaient que peu des valeurs moyennes. Les valeurs maximales et minimales ne sont pas mentionnées.

Les compositions des lactosérums employés pour l'une et pour l'autre série d'essais ne différaient que peu.

Des essais préliminaires ont indiqué que des diminutions de 80 p. 100 et 95 p. 100 de la conductivité du lactosérum correspondaient à peu de chose près à une élimination de respectivement 70 p. 100 et 90 p. 100 de la quantité initiale de cendre; nous nous sommes donc basés sur la diminution de la conductivité pour arrêter les essais. Il ressort des données du tableau 1 que nous avons approché notre objectif de fort près dans la première série d'essais, tandis que seulement 88,4 p. 100 de la quantité initiale de cendre ont été éliminés dans la deuxième série, au lieu des 90 p. 100 désirés.

Le transport du "solvant " atteignait 3,8 p. 100 dans la déminéralisation à 70 p. 100 ; en d'autres termes, près de 3,8 p. 100 de la 


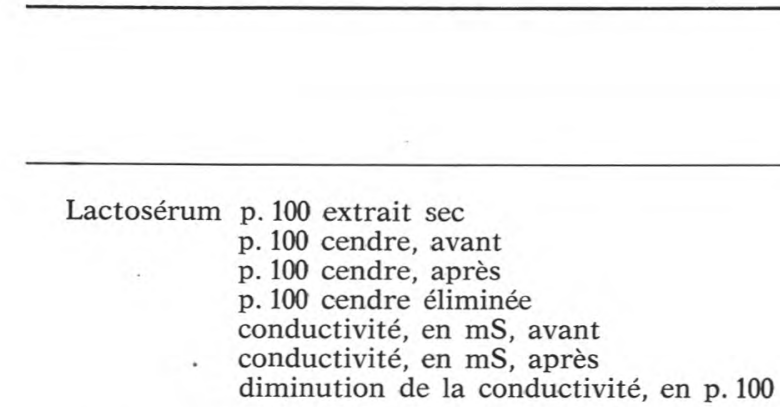

Transport de «solvant», en p. 100

Densité de courant, en $\mathrm{mA} / \mathrm{cm}^{2}$

après diminution de la conductivité de

50 p. 100

60 p. 100

70 p. 100

80 p. 100

90 p. 100

95 p. 100

Consommation de courant continu, en Wh

après diminution de la conductivité de

50 p. 100
60 p. 100

70 p. 100

80 p. 100

90 p. 100

95 p. 100

Litres de lactosérum traités par $\mathrm{m}^{2}$ de membrane et par heure après diminution de la conductivité de

50 p. 100

\section{5,3}

2,04

0,64

70,0

12,45
2,90

79,9

3,8

25,0

18,5

12,0
8,7

8,7

60 p. 100

70 p. 100

80 p. 100

90 p. 100

95 p. 100

74

104

140

184

72

57

41

28
25,0

2,03

0,26

88,4

12,53

0,62

95,1

7,5

24,1

17,4

11,2

4,6
2,9

76

106

143

187

261

341

444

Cendre éliminée par $\mathrm{m}^{2}$ de membrane et par heure, en gramme

Consommation de courant continu par gramme de cendre éliminée, en Wh 
quantité de lactosérum traitée ont été retrouvés dans le concentré. Dans la déminéralisation à 90 p. 100 , on arrivait presque au double : 7,5 p. 100.

Malgré la tension constante, la densité de courant était à tout moment un peu moindre dans la deuxième série d'essais que dans la première. Ceci a eu pour conséquence que la durée du traitement requis pour obtenir une diminution de 80 p. 100 de la conductivité a été un peu plus longue et la consommation de courant continu un peu plus élevée. De ce fait, la diminution de 80 p. 100 de la conductivité qui a pu être obtenue pour 281 de lactosérum par $\mathrm{m}^{2}$ de membrane et par heure dans la première série d'essais, n'a pu être obtenue que pour 261 dans la seconde série. Comme pratiquement tous les paramètres étaient faciles à régler et étaient aussi contrôlés efficacement, la cause doit être cherchée dans les minimes différences en composition des lactosérums et dans l'efficacité du nettoyage.

Le calcul de la consommation de courant continu a été basé uniquement sur la tension par paire de cellules (obtenue par mesurage direct entre 5 paires de cellules). L'indication de la consommation calculée d'après la tension aux électrodes n'a guère de sens ici.

La durée moyenne d'une déminéralisation à 90 p. 100 a été de $2 \mathrm{~h} 12 \mathrm{mn}$ et correspond à la durée d'un batch recommandée par certains constructeurs pour limiter la prolifération des bactéries. La déminéralisation à 70 p. 100 n'a exigé que $34 \mathrm{mn}$.

On peut aisément déduire des données du tableau 1 que les frais diffèrent considérablement selon que l'on élimine 70 p. 100 ou 90 p. 100 de la quantité initiale de cendre. Si la quantité de lactosérum déminéralisée à 70 p. 100 s'est élevée à 281 par heure et par $\mathrm{m}^{2}$ de membrane, la quantité déminéralisée à 90 p. 100 n'atteignait plus que 81 . Dans le premier cas on avait éliminé $444 \mathrm{~g}$ de cendre par heure et par $\mathrm{m}^{2}$ de membrane contre seulement $145 \mathrm{~g}$ dans la deuxième série d'essais. Cette différence influe naturellement dans une très large mesure sur les frais d'investissement.

La consommation de courant continu par kg d'extrait sec déminéralisé s'est élevée pour la déminéralisation à 90 p. 100 à exactement le double de la consommation relevée pour la déminéralisation à 70 p. 100 .

Une température élevée est favorable à l'efficience du courant continu. Certains constructeurs conseillent de ne pas opérer à plus de $30^{\circ} \mathrm{C}$ quand la densité de courant est grande lors de la mise en marche. Au cours de nos essais effectués à $45^{\circ} \mathrm{C}$, la densité de courant maximale atteignait $25 \mathrm{~mA} / \mathrm{cm}^{2}$; l'efficience du courant nécessaire pour l'obtention d'un degré de déminéralisation déterminé est restée pratiquement inchangée malgré le nombre croissant d'essais et les membranes n'ont subi aucun dommage visible. 
En augmentant la tension par paire de cellules et (ou) la vitesse linéaire, on peut augmenter la densité de courant jusqu'à un certain point sans que des phénomènes de polarisation néfaste se produisent de façon nettement décelables. Les frais d'investissement peuvent être quelque peu comprimés de cette manière. Nous sommes toutefois convaincus que les économies ainsi réalisées ne contribueraient que dans une mesure très restreinte à une réduction des frais totaux, ceci pour différentes raisons (entre autres l'abrègement de la longévité des membranes et une augmentation éventuelle de la consommation de courant alternatif).

\subsection{Données d'analyse}

Les principales données relatives à l'analyse du lactosérum sont rassemblées dans le tableau 2. Toutes les teneurs y sont exprimées en pourcentage de poids. Aucun composant formant des précipitations n'a été décelé dans aucun essai.

TABLEAU 2. - Résultats moyens de l'analyse

\begin{tabular}{|c|c|c|c|}
\hline & & $\begin{array}{l}\text { Elimination } \\
70 \text { p. } 100 \mathrm{de} \\
\text { cendre initiale }\end{array}$ & $\begin{array}{l}\text { Elimination } \\
90 \text { p. } 100 \text { de } \\
\text { cendre initiale }\end{array}$ \\
\hline $\mathrm{Kg}$ lactosérum & $\begin{array}{l}\text { avant } \\
\text { après } \\
\text { perte, en p. } 100\end{array}$ & $\begin{array}{c}16,65 \\
15,87 \\
4,7\end{array}$ & $\begin{array}{c}16,62 \\
15,19 \\
8,6\end{array}$ \\
\hline Extrait sec & $\begin{array}{l}\text { p. } 100 \text { avant } \\
\text { p. } 100 \text { après } \\
\text { perte, en p. } 100\end{array}$ & $\begin{array}{c}25,300 \\
24,400 \\
8,1\end{array}$ & $\begin{array}{l}25,000 \\
23,900 \\
12,6\end{array}$ \\
\hline Azote total & $\begin{array}{l}\text { p. } 100 \text { avant } \\
\text { p. } 100 \text { après } \\
\text { perte, en p. } 100\end{array}$ & $\begin{array}{l}0,528 \\
0,519 \\
6,8\end{array}$ & $\begin{array}{l}0,544 \\
0,538 \\
8,9\end{array}$ \\
\hline Azote non protéique & $\begin{array}{l}\text { p. } 100 \text { avant } \\
\text { p. } 100 \text { après } \\
\text { perte, en p. } 100\end{array}$ & $\begin{array}{l}0,161 \\
0,142 \\
14,8\end{array}$ & $\begin{array}{l}0,167 \\
0,139 \\
25,0\end{array}$ \\
\hline Azote protéique & $\begin{array}{l}\text { p. } 100 \text { avant } \\
\text { p. } 100 \text { après } \\
\text { perte, en p. } 100\end{array}$ & $\begin{array}{l}0,367 \\
0,377 \\
1,6\end{array}$ & $\begin{array}{l}0,377 \\
0,139 \\
3,2\end{array}$ \\
\hline Lactose (monohydrate) & $\begin{array}{l}\text { p.100 avant } \\
\text { p. } 100 \text { après } \\
\text { perte, en p. } 100\end{array}$ & $\begin{array}{l}19,820 \\
20,038 \\
3,6\end{array}$ & $\begin{array}{c}19,422 \\
20,263 \\
4,7\end{array}$ \\
\hline Acide citrique (anhydride) & $\begin{array}{l}\text { p. } 100 \text { avant } \\
\text { p. } 100 \text { après } \\
\text { perte, en p. } 100\end{array}$ & $\begin{array}{l}0,601 \\
0,555 \\
12,0\end{array}$ & $\begin{array}{l}0,590 \\
0,158 \\
75,5\end{array}$ \\
\hline
\end{tabular}




\begin{tabular}{|c|c|c|c|}
\hline & & $\begin{array}{l}\text { Elimination } \\
70 \text { p. } 100 \mathrm{de} \\
\text { cendre initiale }\end{array}$ & $\begin{array}{l}\text { Elimination } \\
\text { de } 90 \text { p. } 100 \\
\text { cendre initiale }\end{array}$ \\
\hline Cendre & $\begin{array}{l}\text { p. } 100 \text { avant } \\
\text { p. } 100 \text { après } \\
\text { élimination, en p. } 100\end{array}$ & $\begin{array}{l}2,040 \\
0,640 \\
70,0\end{array}$ & $\begin{array}{l}2,030 \\
0,260 \\
88,4\end{array}$ \\
\hline $\mathrm{Na}$ & $\begin{array}{l}\text { p. } 100 \text { avant } \\
\text { p. } 100 \text { après } \\
\text { élimination, en p. } 100\end{array}$ & $\begin{array}{l}0,252 \\
0,082 \\
69,1\end{array}$ & $\begin{array}{l}0,253 \\
0,020 \\
92,9\end{array}$ \\
\hline K & $\begin{array}{l}\text { p. } 100 \text { avant } \\
\text { p. } 100 \text { après } \\
\text { élimination, en p. } 100\end{array}$ & $\begin{array}{l}0,715 \\
0,113 \\
84,9\end{array}$ & $\begin{array}{l}0,722 \\
0,007 \\
99,2\end{array}$ \\
\hline $\mathrm{Ca}$ & $\begin{array}{l}\text { p. } 100 \text { avant } \\
\text { p. } 100 \text { après } \\
\text { élimination, en p. } 100\end{array}$ & $\begin{array}{l}0,192 \\
0,050 \\
75,0\end{array}$ & $\begin{array}{l}0,205 \\
0,033 \\
85,3\end{array}$ \\
\hline $\mathrm{Cl}$ & $\begin{array}{l}\text { p. } 100 \text { avant } \\
\text { p. } 100 \text { après } \\
\text { élimination, en p. } 100\end{array}$ & $\begin{array}{l}0,431 \\
0,006 \\
98,6\end{array}$ & $\begin{array}{l}0,408 \\
0,007 \\
98,5\end{array}$ \\
\hline$P$ & $\begin{array}{l}\text { p. } 100 \text { avant } \\
\text { p. } 100 \text { après } \\
\text { élimination, en p. } 100\end{array}$ & $\begin{array}{c}0,185 \\
0,089 \\
54,8\end{array}$ & $\begin{array}{l}0,175 \\
0,031 \\
82,8\end{array}$ \\
\hline Titre d'acidité en ${ }^{\circ} \mathrm{N}$ & $\begin{array}{l}\text { avant } \\
\text { après } \\
\text { baisse }\end{array}$ & $\begin{array}{l}47 \\
35 \\
12\end{array}$ & $\begin{array}{l}45 \\
34 \\
11\end{array}$ \\
\hline Acidité, en meq & $\begin{array}{l}\text { avant } \\
\text { après } \\
\text { diminution, en p. } 100\end{array}$ & $\begin{array}{l}705 \\
505 \\
28,4\end{array}$ & $\begin{array}{l}675 \\
472 \\
30,1\end{array}$ \\
\hline $\mathrm{pH}$ & $\begin{array}{l}\text { avant } \\
\text { après } \\
\text { baisse }\end{array}$ & $\begin{array}{l}6,15 \\
5,76 \\
0,39\end{array}$ & $\begin{array}{l}6,25 \\
4,87 \\
1,38\end{array}$ \\
\hline
\end{tabular}

La déminéralisation à 70 p. 100 est allée de pair avec une perte moyenne de 4,7 p. 100 du nombre initial de $\mathrm{kg}$ de lactosérum ; cette perte s'est élevée à 8,6 p.100 dans la déminéralisation à 90 p. 100 . Cette différence est évidemment imputable en partie à la différence en transport du « solvant».

Le transport du "solvant " a aussi pour conséquence que les teneurs en certains composants du lactosérum, tels que l'azote total, l'azote protéique et le lactose, ne sont pas beaucoup plus basses ou sont parfois plus élevées après la déminéralisation qu'avant celle-ci.

La déminéralisation à 70 p. 100 a entraîné une perte de 8,1 p. 100 de l'extrait sec initial ; cette perte s'est élevée à 12,6 p. 100 dans 
la déminéralisation à $90 \mathrm{p} .100$. Ces pertes ne sont imputables qu'en partie à l'élimination de la cendre. Dans la déminéralisation à 70 p. 100 , la quantité d'extrait sec perdue était constituée pour 70 p. 100 de cendre et pour 30 p. 100 d'autres composants du lactosérum ; les 8,1 p. 100 d'extrait sec perdus se décomposent donc en environ 5,7 p. 100 de cendre et 2,4 p. 100 d'autres composants. La perte en extrait sec après déminéralisation à $90 \mathrm{p} .100$ ne comportait que 57 p. 100 de cendre et 43 p. 100 d'autres composants du lactosérum, de sorte que les 12,6 p. 100 d'extrait sec perdus se décomposent en environ 7,2 p 100 de cendre et 5,4 p. 100 d'autres composants.

Les pertes d'azote non protéique se sont élevées à respectivement 14,8 p. 100 et 25 p. 100 , mais les pertes d'azote protéique à seulement 1,6 p. 100 et 3,2 p. 100 . La quantité totale d'azote perdue pendant la déminéralisation à 70 p. 100 était constituée de 77 p. 100 d'azote non protéique et de 23 p. 100 d'azote protéique; une répartition à peu près identique a été constatée pour la déminéralisation à 90 p. 100. On peut admettre que la plus grande partie des protéines perdues ne se retrouve pas dans le concentré, mais est retenue dans les cellules à lactosérum. En valeur absolue, la perte de protéines (azote protéique $\times 6,38$ ) au cours des essais a naturellement été minime : $8 \mathrm{~g}$ et $13 \mathrm{~g}$ pour les déminéralisations à respectivement 70 p. 100 et 90 p. 100 .

L'élimination des ions, exprimée en pourcentage, concorde à peu près avec ce que l'on peut escompter d'après la théorie générale. Les quantités d'ions $\mathrm{Ca}$ éliminées sont relativement élevées, aussi bien après 70 p. 100 qu'après 90 p. 100 de déminéralisation, mais ceci est peut-être imputable à la présence de $\mathrm{CaCl}_{2}$ (ajouté pendant la fabrication du fromage). Seule l'élimination des ions $\mathrm{Na}$ s'écarte assez sensiblement des prévisions. En théorie, les ions $\mathrm{K}$ et $\mathrm{Cl}$ sont plus facilement éliminés que les ions $\mathrm{Na}$, mais l'élimination de seulement 93 p. 100 des ions $\mathrm{Na}$ après une déminéralisation à 90 p. 100 est plutôt faible par rapport aux quelques 99 p. 100 atteints pour les ions $\mathrm{K}$ et $\mathrm{Cl}$. De plus, seulement quelques 69 p. 100 des ions $\mathrm{Na}$ étaient éliminés après la déminéralisation à $70 \mathrm{p} .100$. Aucune explication satisfaisante de ce phénomène ne peut, provisoirement, être donnée.

Environ 28 p. 100 du nombre initial de meq d'acides $\left(1^{\circ} \mathrm{N}\right.$ indique 1 meq d'acide par litre) étaient éliminés après la déminéralisation à 70 p. 100 . On peut admettre que quasiment pas de lactose n'a été converti en acide lactique au cours de cette série d'essais : lactosérum concentré à nombre de germes peu élevé, électrodialyse à $45^{\circ} \mathrm{C}$, durée moyenne de seulement $34 \mathrm{mn}$. Après $90 \mathrm{p} .100$ de déminéralisation, il restait encore dans le lactosérum 70 p. 100 du nombre initial de meq d'acide. Il est difficile de déterminer en quelle mesure ceci est imputable à la formation d'acide lactique pendant l'électrodialyse (durée moyenne de $132 \mathrm{mn}$ ). D'une part, 
environ 75 p. 100 de l'acide citrique étaient éliminés après la déminéralisation à $90 \mathrm{p} .100$ (contre seulement $12 \mathrm{p} .100$ après $70 \mathrm{p} .100$ de déminéralisation) et, d'autre part, le minime écart en pourcentage d'élimination du lactose entre les déminéralisations à 70 p. 100 et à 90 p. 100 peut aisément s'expliquer par la grande différence en transport du "solvant », lequel contient naturellement une partie des composants du lactosérum.

\section{VI. - ESTIMATION DU COUT DE L’ELECTRODIALYSE}

Les teneurs en extrait $\mathrm{sec}$ et en cendre du lactosérum varient selon la saison et, surtout, selon le mode de fabrication du fromage (par exemple : quantités de $\mathrm{KNO}_{3}$ et de $\mathrm{CaCl}_{2}$, composition de l'eau, degré de dilution du lactosérum).

Le lactosérum employé pour nos essais provenait de la laiterie expérimentale et contenait environ 5,43 p. 100 d'extrait sec et 0,44 p. 100 de cendre. L'analyse de lactosérums provenant d'un certain nombre de laiteries industrielles a démontré que les fabricants de poudre peuvent compter recevoir un lactosérum d'une densité moyenne de 1,0215 et à teneurs en extrait sec et en cendre de respectivement 5,4 p. 100 et 0,5 p. 100 .

Nous nous proposons de calculer ici le coût de l'élimination quotidienne de respectivement 70 p. 100 et 90 p. 100 de la quantité initiale de cendre de 100000 l d'un lactosérum du type précité. Ceci en supposant que l'on utilise les mêmes paramètres que dans les traitements expérimentaux.

Le tableau 3 donne un aperçu des conditions valables pour les calculs.

TABLEAU 3. - Capacité de l'installation

1000001 de lactosérum par jour

Lactosérum : mélange du premier et du deuxième sérums provenant de la fabrition de fromage Gouda

densité 1,0215 - titre d'acidité $10^{\circ} \mathrm{N}-\mathrm{pH} 6,50$

extrait sec 5,4 p. 100

cendre 0,5 p. 100

Déminéralisation : élimination de 70 p. 100 ou de 90 p. 100 de la quantité initiale de cendre

Paramètres : comme dans les traitements expérimentaux 


\subsection{Facteurs de base pour le calcul des frais indirects}

Le tableau 4 donne un aperçu des facteurs de base pour le calcul des frais indirects.

TABLEAU 4. - Facteurs de base pour le calcul des frais indirects

\begin{tabular}{|c|c|c|}
\hline & $\begin{array}{l}\text { Elimination } \\
70 \text { p. } 100 \\
\text { de cendre }\end{array}$ & $\begin{array}{l}\text { Elimination } \\
90 \mathrm{p} .100 \\
\text { de cendre }\end{array}$ \\
\hline $\begin{array}{l}\text { Nombre de modules WD } 10 / 4 \text { à } 53 \text { paires de cellules } \\
\text { Nombre de modules WD } 10 / 4 \text { à } 54 \text { paires de cellules }\end{array}$ & 3 & 12 \\
\hline $\begin{array}{l}\text { Estimation des frais d'investissement (membranes } \\
\text { non comprises), en FB }\end{array}$ & 5000000 & 12000000 \\
\hline $\begin{array}{l}\text { Amortissement, en années } \\
\text { Intérêt pour privation de capital, en p. } 100 \\
\text { Assurance et divers, en p. } 100 \text { de l'investissement }\end{array}$ & & $\begin{array}{l}5 \\
6 \\
2\end{array}$ \\
\hline
\end{tabular}

Werkspoor Water met sur le marché des modules du type WD $10 / 4$, dont l'exécution standard contient 50 paires de cellules totalisant $14 \mathrm{~m}^{2}$ de surface de membrane active.

Nous supposons que l'on travaille $24 \mathrm{~h}$ par jour : $18 \mathrm{~h}(3 \times 6 \mathrm{~h})$ sont dévolues à la déminéralisation et $6 \mathrm{~h}(3 \times 2 \mathrm{~h})$ au nettoyage. Afin de freiner la prolifération des bactéries, la déminéralisation s'effectue par batches de 2 h ( 3 batches consécutifs, entrecoupés d'un bref rinçage).

Les $100000 \mathrm{l}$ de lactosérum contiennent $510,75 \mathrm{~kg}$ de cendre, de sorte que la déminéralisation à 70 p. 100 doit en éliminer $19863 \mathrm{~g}$ par heure et celle à 90 p. 10025538 g par heure. Selon les données du tableau 1 ceci nécessite respectivement environ 44,8 et $176,2 \mathrm{~m}^{2}$ de membranes, soit 3,2 et 12,6 modules standards, selon que l'on veut déminéraliser de 70 p. 100 ou de 90 p. 100 . Le nombre de paires de cellules par module peut être augmenté dans une certaine mesure ; si on prend respectivement 3 modules à 54 paires de cellules et 12 modules à 53 paires on arrive largement au nombre requis de $\mathrm{m}^{2}$ de membranes.

Les frais d'investissement, membranes non comprises, pour une installation automatique peuvent être estimés à 5000000 FB pour une déminéralisation à 70 p. 100 et à $12000000 \mathrm{FB}$ pour une déminéralisation à 90 p. 100. L'investissement comprend : toutes les conduites électriques et hydrauliques internes, les pompes, les bacs de cir- 
TABLEAU 5. - Facteurs de base pour le calcul des frais directs

Schéma de fonctionnement Nombre de jours par an

Nombre d'heures par jour

Nombre d'heures par jour destinées à la déminéralisation

Membranes

Nombre de membranes

Prix moyen d'une membrane, en FB

Remplacement des membranes

Electricité

Consommation de courant continu, en Wh par gramme de cendre éliminée, électrodes comprises

Marge de sécurité, en p. 100, pour la consommation de courant continu

Consommation de courant alternatif, en p. 100 de la consommation de

courant continu

Prix du kWh, en FB

Eau

Consommation, en $\mathrm{m}^{3}$ par jour

Prix du $\mathrm{m}^{3}$, en $\mathrm{FB}$

Produits chimiques

Consommation de $\mathrm{HCl}$ à $35 \mathrm{p} .100$, en $\mathrm{kg}$ par jour

Consommation de $\mathrm{NaOH}$ à $30 \mathrm{p} .100$, en $\mathrm{kg}$ par jour

Prix du $\mathrm{HCl}$ à 35 p. 100 , en $\mathrm{FB} / \mathrm{kg}$

Prix du $\mathrm{NaOH}$ à $30 \mathrm{p} .100$, en $\mathrm{FB} / \mathrm{kg}$

Coût d'autres produits chimiques, en p. 100 du coût total en $\mathrm{HCl}$ et

$\mathrm{NaOH}\left(\mathrm{Na}_{2} \mathrm{SO}_{4}, \mathrm{H}_{2} \mathrm{SO}_{4}, \mathrm{NaCl}\right.$, nettoyage externe)

Main-d'œuvre

Nombre d'heures - homme par jour

Coût par heure-homme, en FB

\section{Entretien}

Production

Frais d'entretien, en p. 100 de l'investissement

Perte d'extrait sec, en p. 100

Pourcentage d'eau de la poudre de lactosérum déminéralisé

Nombre de $\mathrm{kg}$ de poudre de lactosérum déminéralisé, par an

\begin{tabular}{|c|c|}
\hline $\begin{array}{l}\text { Elimination } \\
\text { de } 70 \text { p. } 100 \\
\text { de cendre }\end{array}$ & $\begin{array}{l}\text { Elimination } \\
\text { de } 90 \text { p. } 100 \\
\text { de cendre }\end{array}$ \\
\hline \multicolumn{2}{|c|}{$\begin{array}{r}250 \\
24 \\
18\end{array}$} \\
\hline \multicolumn{2}{|c|}{$\begin{array}{c}500 \\
\text { tous les ans }\end{array}$} \\
\hline 0,87 & 1,26 \\
\hline & 1 \\
\hline 30 & 60 \\
\hline $\begin{array}{l}40 \\
30\end{array}$ & $\begin{array}{l}70 \\
50\end{array}$ \\
\hline & 5 \\
\hline 3 & 60 \\
\hline & 1 \\
\hline 8,3 & 12,8 \\
\hline 1303600 & 1239700 \\
\hline
\end{tabular}


culation du lactosérum, du concentré, du liquide de rinçage des électrodes et des solutions de nettoyage et les réservoirs à produits chimiques concentrés. Il n'est pas tenu compte ici de frais relatifs au bâtiment.

Les autres facteurs figurant au tableau 3 ne nécessitent pas d'explications.

\subsection{Facteurs de base pour le calcul des frais directs}

Le tableau 5 donne un aperçu des facteurs de base pour le calcul des frais directs. Nous ne commenterons ici que ceux qui nécessitent quelques explications.

Les opinions diffèrent quant à la longévité des membranes. Celle des membranes cationiques peut être estimée à 1 ou 2 ans ; celle des membranes anioniques est moins grande. Il est sage de prévoir un remplacement annuel dans le calcul des frais ; c'est pourquoi le coût des membranes ne figure pas parmi les frais indirects. Les membranes anioniques sont sensiblement plus chères que les cationiques ; pour le module choisi le prix moyen est d'environ 500 FB.

D'après le tableau 1 il faut compter 0,79 ou 1,15 Wh en courant continu, selon que l'on déminéralise à 70 p. 100 ou à 90 p. 100 , pour éliminer $1 \mathrm{~g}$ de cendre. Puisqu'il faut tenir compte ici de la tension mesurée entre les électrodes, la consommation totale est estimée à respectivement 0,87 et $1,26 \mathrm{Wh}$. La consommation de courant continu ainsi calculée est majorée de 10 p. 100 par mesure de prudence. Cette majoration n'influe que peu sur les frais totaux, comme le montre la récapitulation des frais (tab. 6).

En décrivant les conditions de travail nous avons signalé que la chute de pression n'était que de $0,25 \mathrm{~kg} / \mathrm{cm}^{2}$ pour le module WD $6 / 2$. C'est là une valeur très basse et la consommation de courant alternatif ne s'élèvera, au plus, qu'à 50 p. 100 de la consommation de courant continu pour le module WD 10/4.

Malgré l'automatisation, il faut compter 3 ou $5 \mathrm{~h}$-homme par jour selon que l'on déminéralise à 70 p. 100 ou à 90 p. 100 . Ceci en comprenant le grand nombre d'heures-homme requises pour le remplacement " annuel " des membranes et pour le remplacement à des intervalles irréguliers d'une ou plusieurs membranes endommagées (ce qui nécessite également l'ouverture de tout le paquet). Il faut aussi tenir compte du nettoyage externe et de l'entretien de l'installation, de l'exécution de petites réparations, des différents travaux hors la mise en marche et de l'arrêt, ainsi que d'un minimum de surveillance pendant le fonctionnement.

Le tableau 2 montre que les pertes d'extrait sec se sont élevées à $8,1 \mathrm{p} .100$ et $12,6 \mathrm{p} .100$ pour les déminéralisations à respectivement 70 p. 100 et 90 p. 100 . Le calcul des frais table, par prudence, sur des pertes de respectivement 8,3 p. 100 et 12,8 p. 100 . 
TABLEAU 6. - Récapitulation des frais, en FB

\begin{tabular}{|c|c|c|c|c|c|c|c|c|c|c|}
\hline & \multicolumn{2}{|c|}{ Frais totaux } & \multicolumn{2}{|c|}{$\begin{array}{l}\text { Frais par } \mathrm{kg} \\
\text { de poudre }\end{array}$} & \multicolumn{3}{|c|}{$\begin{array}{l}\text { Elimination de } 70 \text { p. } 100 \\
\text { de cendre }\end{array}$} & \multicolumn{3}{|c|}{$\begin{array}{l}\text { Elimination de } 90 \text { p. } 100 \\
\text { de cendre }\end{array}$} \\
\hline & \multirow{2}{*}{$\begin{array}{c}\text { élimina- } \\
\text { tion de } \\
70 \mathrm{p} .100 \\
\text { de } \\
\text { cendre }\end{array}$} & \multirow{2}{*}{$\begin{array}{l}\text { élimina- } \\
\text { tion de } \\
90 \mathrm{p} .100 \\
\text { de } \\
\text { cendre }\end{array}$} & \multirow{2}{*}{$\begin{array}{c}\text { élimina- } \\
\text { tion de } \\
70 \text { p. } 100 \\
\text { de } \\
\text { cendre }\end{array}$} & \multirow{2}{*}{$\begin{array}{c}\text { élimina- } \\
\text { tion de } \\
90 \text { p. } 100 \\
\text { de } \\
\text { cendre }\end{array}$} & \multicolumn{3}{|c|}{ part en p. 100 dans les } & \multicolumn{3}{|c|}{ part en p. 100 dans les } \\
\hline & & & & & $\begin{array}{c}\text { frais } \\
\text { indirects }\end{array}$ & $\begin{array}{l}\text { frais } \\
\text { directs }\end{array}$ & $\begin{array}{l}\text { frais } \\
\text { totaux }\end{array}$ & $\begin{array}{l}\text { frais } \\
\text { indirects }\end{array}$ & $\begin{array}{l}\text { frais } \\
\text { directs }\end{array}$ & $\begin{array}{l}\text { frais } \\
\text { totaux }\end{array}$ \\
\hline Amortissement & 1000000 & 2400000 & 0,767 & 1,936 & 71,4 & & 51,5 & 71,4 & & 51,0 \\
\hline capital & 300000 & 720000 & 0,230 & 0,581 & 21,4 & & 15,5 & 21,4 & & 15,3 \\
\hline Assurance et divers & 100000 & 240000 & 0,077 & 0,194 & 7,2 & & 5,1 & 7,2 & & 5,1 \\
\hline Total des frais indirects & 1400000 & 3360000 & 1,074 & 2,711 & & & 72,1 & & & 71,4 \\
\hline Remplacement de membranes & 162000 & 636000 & 0,125 & 0,513 & & 30,0 & 8,3 & & 47,3 & 13,5 \\
\hline Electricité & 141000 & 263000 & 0,108 & 0,212 & & 26,1 & 7,3 & & 19,6 & 5,6 \\
\hline Eau & 30000 & 60000 & 0,023 & 0,048 & & 5,5 & 1,5 & & 4,5 & 1,3 \\
\hline Produits chimiques & 38000 & 64000 & 0,029 & 0,052 & & 7,0 & 2,0 & & 4,8 & 1,4 \\
\hline Main-d'œuvre & 120000 & 120000 & 0,092 & 0,161 & & 22,2 & 6,2 & & 14,9 & 4,2 \\
\hline Entretien & 50000 & 120000 & 0,038 & 0,097 & & 9,2 & 2,6 & & 8,9 & 2,6 \\
\hline Total des frais directs & 541000 & 1343000 & 0,415 & 1,083 & & & 27,9 & & & 28,6 \\
\hline Frais totaux & 1941000 & 4703000 & 1,489 & 3,794 & & & & & & \\
\hline
\end{tabular}




\subsection{Récapitulation des frais}

Le tableau 6 indique les frais totaux, les frais par $\mathrm{kg}$ de poudre de lactosérum déminéralisé et donne un aperçu de la ventilation des frais.

Le coût total d'une déminéralisation à 90 p. 100 est environ 2,4 fois plus élevé que celui d'une déminéralisation à 70 p. 100, les frais indirects représentant dans les deux cas près de 70 p. 100 du coût total.

La perte d'extrait sec étant plus grande dans la déminéralisation à 90 p. 100 que dans celle à 70 p. 100 , le total des frais au kg de poudre déminéralisée à 90 p. 100 est environ 2,5 fois plus élevé que le total des frais au $\mathrm{kg}$ de poudre déminéralisée à 70 p. 100 .

Le remplacement de membranes représente environ 30 p. 100 et 47 p. 100 des frais directs, mais seulement quelques 8 p. 100 et 13 p. 100 des frais totaux, selon le taux de déminéralisation. Parmi les frais directs, la consommation d'électricité figure en deuxième et la main-d'œuvre en troisième place. Leur part dans les frais totaux n'est pas bien élevée.

\section{VII. - CONCLUSION}

Le module-pilote WD $6 / 2$ testé est pratiquement construit de la même façon que le module industriel WD $10 / 4$, de sorte que les résultats expérimentaux se prêtent remarquablement à l'extrapolation.

Malgré la haute température de service et le grand nombre d'essais, l'efficience de courant est restée à peu près inchangée pour chaque degré de déminéralisation choisi. Ni les membranes, ni les électrodes n'ont subi de détériorations nettement décelables. La densité de courant n'a toutefois jamais atteint des valeurs élevées (maximum $25 \mathrm{~mA} / \mathrm{cm}^{2}$ ). Des phénomènes de polarisation néfastes ne se sont pas produits et le schéma de nettoyage a donné satisfaction.

Après 70 p. 100 et 90 p. 100 de déminéralisation, la cendre représentait respectivement 70 p. 100 et 57 p. 100 de la quantité d'extrait sec perdue. Quand on déminéralisait à 90 p. 100, les pertes d'acide citrique, d'azote non protéique, de protéines (azote protéique $\times 6,38$ ) et de lactose étaient plus élevées de respectivement 500 p. 100, 65 p. 100 , 50 p. 100 et 25 p. 100 que quand on déminéralisait à 70 p. 100. Ces différences sont imputables en partie au transport du "solvant " (lequel contient aussi des composants du lactosérum), plus intense pour un degré de déminéralisation élevé.

Le coût total d'une déminéralisation à 70 p. 100 est plutôt modeste, mais celui d'une déminéralisation à 50 p. 100 est assez élevé : pour une installation d'une capacité déterminée et fonctionnant dans des conditions expérimentales, nous sommes arrivés, en 
incluant un remplacement annuel des membranes, à respectivement environ 1,5 et 3,8 FB par $\mathrm{kg}$ de poudre déminéralisée. Une déminéralisation à 90 p. 100 par électrodialyse ne pose toutefois pas de problèmes et les frais directs (environ 1,1 FB par $\mathrm{kg}$ de poudre déminéralisée pour l'installation et les conditions de travail en cause ici) sont très acceptables. Les frais directs de la déminéralisation à 70 p. 100 sont très bas (environ 0,4 FB par $\mathrm{kg}$ de poudre déminéralisée).

\section{Rés u mé}

L'offre de poudre de lactosérum augmentera à mesure que la surveillance de la pollution deviendra plus rigoureuse et ceci aura une influence défavorable sur le prix. Il faut donc chercher d'autres moyens de valoriser une importante quantité de lactosérum. La déminéralisation est un de ces moyens et l'on constate un accroissement de la demande de poudre de lactosérum déminéralisée.

Une discussion des données relatives à l'électrodialyse et à l'analyse du lactosérum, obtenues au cours de deux séries d'essais visant à déminéraliser de respectivement 70 p. 100 et 90 p. 100, est suivie d'une évaluation des frais de déminéralisation, basée sur les résultats des essais.

Les essais ont été effectués avec un module WD 6/2 de la firme néerlandaise Werkspoor Water équipé de membranes Selemion des types CMV et AMV de la firme japonaise Asahi Glass. Les principales conditions de travail étaient : lactosérum à 25 p. 100 d'extrait sec et 2 p. 100 de cendre, température de $45^{\circ} \mathrm{C}$, vitesse linéaire de $10 \mathrm{~cm} / \mathrm{s}$, densité maximale du courant $25 \mathrm{~mA} / \mathrm{cm}^{2}$, tension constante de $2,5 \mathrm{~V}$ par paire de cellules.

Une déminéralisation à 70 p. 100 a éliminé 444 g et une déminéralisation à 90 p. 100 n'a éliminé que $145 \mathrm{~g}$ de cendre par heure et par $\mathrm{m}^{2}$ de membrane. La consommation de courant continu s'est élevée à 0,79 Wh par gramme dans le premier cas et à 1,15 Wh dans le second; pour le calcul on s'est servi de la tension par paire de cellules (mesure directe entre 5 paires de cellules).

La perte d'extrait sec s'est élevée à 8,1 p. 100 dans la déminéralisation à 70 p. 100 et à 12,6 p. 100 dans celle à 90 p. 100 ; dans le premier cas la quantité d'extrait sec perdue était constituée de 70 p. 100 de cendre et de 30 p. 100 d'autres constituants du lactosérum ; les valeurs correspondantes étaient de 57 p. 100 et de 43 p. 100 dans le second cas. Dans la déminéralisation à 70 p. 100 l'élimination des ions $\mathrm{Na}$ a été moins considérable que normalement à prévoir.

Pour calculer les frais, on part d'une installation capable de déminéraliser à 70 p. 100 ou à 90 p. 100 un volume quotidien de $100000 \mathrm{I}$ 
de lactosérum d'une composition déterminée (e.a. 5,4 p. 100 d'extrait sec et 0,5 p. 100 de cendre) et l'on suppose que l'on utilise les mêmes paramètres qu'au cours des déminéralisations expérimentales. Un aperçu des facteurs de base pour le calcul des frais directs et indirects conduit à une répartition des frais et au prix de revient total. Les frais totaux par $\mathrm{kg}$ de poudre déminéralisée sont plus élevés d'environ 2,5 fois pour la déminéralisation à 90 p. 100 que pour celle à 70 p. 100 : environ $3,8 \mathrm{FB}$ au lieu d'environ 1,5 FB. Les frais indirects représentent environ 70 p. 100 des frais totaux.

\section{A bstract}

The quantity of whey powder offered will increase as pollution control is intensified, and this will reflect unfavourably on the price. Therefore other methods of valorization must be sought for a large portion of the whey. Demineralization is one of the means and the demand for demineralized whey powder is increasing.

A discussion of the data concerning electrodialysis and whey analysis obtained from two experimental series in which a demineralization of respectively 70 p. 100 and 90 p. 100 was aimed at, is followed by an estimate of the cost of demineralization, based on the experimental results.

The experiments were carried out with a module WD $6 / 2$ of the Dutch firm Werkspoor Water, equipped with membranes Selemion types CMV and AMV of the Japanese firm Asahi Glass. The main working conditions were : whey containing 25 p. 100 dry matter and 2 p. 100 ash, temperature $45^{\circ} \mathrm{C}$, linear velocity $10 \mathrm{~cm} / \mathrm{s}$, maximal current density $25 \mathrm{~mA} / \mathrm{cm}^{2}$, constant tension of $2.5 \mathrm{~V}$ per cell pair.

With a 70 p. 100 demineralization $444 \mathrm{~g}$ of ash was removed per h and per $\mathrm{m}^{2}$ of membrane and with a 90 p. 100 demineralization only $145 \mathrm{~g}$. In the first case the consumption of direct current amounted to 0.79 Wh per $\mathrm{g}$ ash removed and in the second case it was $1.15 \mathrm{Wh}$; in the calculation use was made of the tension per cell pair (direct measurement between 5 cell pairs).

With a 70 p. 100 and a 90 p. 100 demineralization respectively 8.1 p. 100 and 12.6 p. 100 dry matter was lost, and in the first case the amount of dry matter lost consisted of 70 p. 100 ash and 30 p. 100 other whey constituents; in the second case these values were 57 and 43 p. 100 respectively. With a 70 p. 100 demineralization fewer $\mathrm{Na}$-ions were removed than would normally be expected.

In calculating the cost we started from an installation which can daily demineralize 100,0001 of whey of a determined composition (i.a. 5.4 p. 100 dry matter and 0.5 p. 100 ash) to 70 p. 100 or to 90 p. 100 ; it is assumed that the same parameters are used as in the experi- 
mental trials. A review of the basic factors for the calculation of the indirect and direct costs, leads to a cost distribution and the total cost. The total costs per $\mathrm{kg}$ demineralized powder are approximately 2.5 times as high for a 90 p. 100 demineralization as for a 70 p. 100 one, approximately 3.8 as compared with approximately $1.5 \mathrm{BF}$. The in direct costs constitute almost 70 p. 100 of the total costs.

\section{Zusammenfassung}

Das Angebot an Molkepulver wird in dem Maße ansteigen, wie der Kampf gegen die Umweltverschmutzung zunimmt, und das wird sich ungünstig auf den Preis auswirken. Für eine große Menge Molke müssen daher andere Valorisationsmittel gefunden werden. Die Entmineralisierung ist eines dieser Mittel, und die Nachfrage nach entsalzenem Molkepulver nimmt zu.

Eine Besprechung der in zwei Versuchsreihen mit Elektrodialyse und Molkenanalyse erhaltenen Daten, wobei eine Entmineralisierung von 70 p. 100 beziehungsweise eine solche von 90 p. 100 ins Auge gefaßt wurde, ist gefolgt von einer auf Grund der Versuchsergebnisse durchgeführten Schätzung der Entsalzungskosten.

Die Versuche wurden mit einem Modul WD 6/2 der holländischen Firma Werkspoor Water durchgeführt, das mit Selemion-Membranen der Type CMV und AMV der japanischen Firma Asahi Glass ausgerüstet war. Die hauptsächlichen Arbeitsbedingungen waren : Molke mit 25 p. 100 Trockensubstanz und 2 p. 100 Asche, Temperatur $45^{\circ} \mathrm{C}$, lineare Geschwindigkeit $10 \mathrm{~cm} / \mathrm{s}$, maximale Stromdichte $25 \mathrm{~mA} / \mathrm{cm}^{2}$, konstante Spannung 2,5 V pro Zellpaar.

Bei 70 p. 100 iger Entmineralisierung wurden pro Stunde und pro $\mathrm{m}^{2}$ Membran $444 \mathrm{~g}$ Asche ausgeschieden, und bei 90 p. 100 iger Entmineralisierung nur 145 g. Im ersten Fall betrug der Verbrauch an Gleichstrom 0,79 Wh pro g ausgeschiedene Asche, und im zweiten Fall 1,15 Wh ; bei der Berechnung benutzte man die pro Zellenpaar herrschende Spannung (direkte Messung zwischen 5 Zellenpaaren).

Bei 70 p. 100 iger und 90 p. 100 iger Entmineralisierung gingen 8,1 bzw. 12,6 p. 100 Trockenmasse verloren, und im ersteren Fall bestand die verlustige Trockenmasse $\mathrm{zu} 70$ p. 100 aus Asche und zu 30 p. 100 aus anderen Molkebestandteilen. Im zweiten Falle betrugen diese Werte 57 bzw. 43 p. 100 . Bei 70 p. 100 iger Entsalzung wurden weniger Na-Ionen ausgeschieden als man normalerweise erwarten würde.

Zwecks Berechnung der Kosten geht man von einer Anlage aus, die täglich 100.000 Liter Molke einer bestimmten Zusammensetzung (u.a. 5,4 p. 100 Trockenmasse und 0,5 p.100 Asche) bis zu 70 p. 100 oder 90 p. 100 entsalzen kann; vorausgesetzt, daß man dieselben Parameter wie bei den Versuchsentmineralisierungen benutzt. Ein Uberblick über 
die - der Berechnung der indirekten und direkten Kosten dienenden - Grundfaktoren führt zu einer Kostenverteilung und zum Gesamtgestehungspreis. Die Gesamtkosten pro $\mathrm{kg}$ entmineralisiertes Molkepulver liegen für eine 90 p. 100 ige Entmineralisierung ca. 2,5 mal höher als für eine solche von 70 p. 100: das sind ca. 3,8 anstatt ca. 1,5 BF. Die indirekten Kosten betragen ungefähr 70 p. 100 der Gesamtkosten.

Reçu pour publication le 30 août 1974.

\section{Références}

Deiss (W. B.) and Kowsoleea (A. R.) (1967). - Emmision-flame photometric determinations of $\mathrm{Na}, \mathrm{K}, \mathrm{Ca}$ and $\mathrm{Mg}$ in plant material and in faeces, urine and milk from cows. Meded. Inst. biol, scheik. Onderz. LandbGew., Jaarb. 1967, Meded. 350, 101.

de Vleeschaumer (A.), Hendrickx (H.) und Heyndrickx (G.) (1948). - Onderzoekingsmethoden van zuivelprodukten. N.V. Standaard-Boekhandel, Antwerpen, p. 56

HCNN (1952). - Bepaling van de normzuurtegraad $\left({ }^{\circ} \mathrm{N}\right)$ van melk. Hoofdcommissie voor de Normalisatie in Nederland, N 913.

IDF (1961). - Determination of the salt content of cheese. International dairy federation, Brussels, International standard FIL-IDF, 17.

IDF (1962). Determination of the total solids content of milk. International dairy federation, Brussels, International standard FIL-IDF, 21.

IDF (1962 a). - Determination of the total nitrogen content of milk by the Kjeldahl method. International dairy federation, Brussels, International standard FIL-IDF, 20.

IDF (1964). - Determination of the ash content of processed cheese products. International dairy federation, Brussels, International standard FIL-IDF. 27.

IDF (1967). - Determination of the citric acid content of cheese and processed cheese products. International dairy federation, Brussels, International standard FIL-IDF, $34 \mathrm{~A}$.

IDF (1967 a). - Determination of the phosphorus content of milk. International dairy federation, Brussels, International standard FIL-IDF, 42.

Triebold (H. O.) and Aurand (L. W.) (1963). - Food composition and analysis. D. Van Nostrand Company, Inc., Princeton, New Jersey, p. 348. 